\title{
MOYAMOYA DISEASE IS ASSOCIATED WITH ENDOTHELIAL ACTIVITY DETECTED BY ANTI-NESTIN ANTIBODY
}

\author{
Jana Chmelova ${ }^{\mathrm{a}, \mathrm{b} *}$, Zdenek Kolara, Vaclav Prochazka ${ }^{\mathrm{b}}$, Romuald Curik ${ }^{\mathrm{c}}$, Jana Dvorackova ${ }^{\mathrm{c}}$, \\ Pavel Sirucek ${ }^{\mathrm{d}}$, Otakar Kraft ${ }^{\mathrm{d}}$, Tomas Hrbac ${ }^{\mathrm{e}}$
}

\author{
a Laboratory of Molecular Pathology, Faculty of Medicine and Dentistry, Palacky University, Olomouc, Czech Republic \\ ${ }^{b}$ Department of Radiology, Faculty of Health Studies and University Hospital, Ostrava, Czech Republic \\ c Department of Pathological Anatomy, Faculty of Health Studies and University Hospital, Ostrava \\ d Department of Nuclear Medicine, University Hospital, Ostrava \\ e Department of Neurosurgery, University Hospital, Ostrava \\ E-mail:jana.chmelova@gmail.com
}

Received: February 8, 2010; Accepted: April 21, 2010

Key words: Moyamoya disease/Nestin/VEGF(R)/Endothelial activation

Aim. To describe the case history and new histopathological findings of a young woman suffering from moyamoya disease.

Methods. The patient underwent brain computed tomography, magnetic resonance imaging and brain angiography. Vessel samples of a. temporalis superficialis were processed by standard histopathological and immunohistochemical methods by analysis of VEGF, VEGFR and nestin expression.

Results. Brain angiography revealed both internal carotid artery stenoses and stenoses of the anterior cerebral arteries. Stenotic parts of vessels were accompanied by coiled and elongated vessels with a picture of „smoke puffs carried away by breeze" after contrast medium application. Histological examination showed: obstruction of lumen, fibrocellular intimal thickening, tortuosity and disruption of internal elastic lamina. Imunohistochemistry confirmed a defect of the internal elastic membrane of the muscular arteria and progressive intimal thickening accompanied by abnormal smooth muscle cells and, VEGF/VEGFR expression in intima. Nestin positivity in endothelium of arteria indicated that endothelial cells are activated.

Conclusion. We found that the endothelium of affected vessels is nestin positive. This, together with the finding of VEGF/VEGFR expression, might suggest an active angiogenetic process We present a new conception of pathogenesis but further studies with higher number of patients are necessary to elucidate the role of these growth factors in the moyamoya disease.

\section{INTRODUCTION}

Moyamoya disease (MMD) is an uncommon disorder with a higher incidence in some countries of East Asia. The incidence of disease is high especially among Japanese and Koreans (0.35-0.94 per 100 000), among Caucasians is very low ( $10 \%$ of that in Japan) (ref. $\left.{ }^{1}\right)$. Two distribution peaks for age are described, the highest peak between 45 and 49 years of age, and the second one between 5 and 9 years of age ${ }^{2}$. MMD is characterized by progressive occlusion of the internal carotid artery and its main branches forming the circle of Willis ${ }^{3}$. The appearence of a vascular network on a cerebral angiogram looks like a puff of cigarette smoke drifting in the air (Fig. 1). For this is reason the disease is called moyamoya (moyamoya means puff of smoke in Japanese). After reduced cerebral perfusion due to arterial stenosis and occlusion, ischemic attacks with other symptoms may occur. Moyamoya disease is usually definitively diagnosed only in the case of bilateral lesions. In cases of unilateral lesions, the diagnosis of moyamoya disease is considered as probable ${ }^{4}$. Moyamoya vessels are dilated perforating arteries with various histopathological changes including fibrin deposits in the wall, fragmented elastic laminae, attenuated media and the formation of microaneurysms. Collapse of the artery lumen and subsequent thrombosis can also be seen ${ }^{4}$. Histopathological findings of the affected arteries include obstruction or stenosis, thickened intima composed of fibrocellular material without lipid deposits or calcifications and extremely tortuosed internal elastic lamina with disruption and decreased number of smooth muscle cells in media ${ }^{5,6}$. It is assumed that proliferation and migration of smooth muscle cells take place in the stenotic or occlusive arterial lesions. According to some authors, the inflammatory process may induce proliferation of smooth muscle cells and this may contribute to the formation of the intracranial occlusive lesions found in MMD. In support of the autoimmune etiology of MMD there are positive findings for the rheumatoid factor and myeloperoxidase-anti-neutrophil cytoplasmic antibody (MPO-ANCA) in the serum of patients with $\mathrm{MMD}^{7}$. Becaus the prevalence of elevated thyroid autoantibodies was higher in the MMD group than in control group, Kim et al. ${ }^{8}$ suggested that immune aberrancies associated with thyroid autoimmunity are also playing a role in developing of this disease. Some epidemiological 
studies implicate infection of head and neck in the development of this disease while others show that genetic factors are contributory too as it is inherited in a polygenic or autosomal dominant mode with a low penetrance ${ }^{7,9,10}$. Since the etiology of MMD is still unknown and the clinical symptoms do not allow for clear differentiation from other conditions such as cerebrovascular, autoimmune, inflammatory and disorders of development, much effort is focussed on description and search for new histopathological markers to confirm the diagnosis. We describe here the case history and new histopathological findings of a young woman suffering from this disease.

\section{CLINICAL HISTORY}

We examined a 31 year old, single, childless woman for a more than one year persisting paresthesia of acral parts of upper limbs, right-hand weakness and double vision. The patient also complained of expressive aphasia. The working diagnosis of the admitting neurologist was ischemic stroke with right-hand hemiparesis, mixed and mostly expressive aphasia, organic inhibition psychosyndrome and cognitive function disorder.

Ultrasound examination revealed bilateral intracranial, internal carotid and anterior cerebral arteries stenoses, normal findings for both vertebral arteries and increased flow velocity in the area of the posterior cerebral arteries. Brain computed tomography (CT) scan and magnetic resonance imaging (MRI) examination showed ischemic changes in left frontal and right parietal lobes. Perfusion CT scan showed reduced blood flow, blood volume and prolonged time-to-peak values in the left hemisphere. Brain angiography verified both internal carotid artery stenoses and stenoses of the anterior cerebral arteries.
The posterior cerebral circulation was normal. In front of stenotic parts, the vessels were elongated and coiled. After filling with contrast media, they created a picture of "smoke puffs carried away by a breeze" (Fig. 1a,1b) that is typical for MMD.

The patient underwent neurosurgery. Anastomosis was performed on the left, between arteria temporalis superficialis and arteria cerebri media supplemented with EDAMS (encephalo-duro-myo-synangiosis). After 4 months, a similar bypass procedure was made on the right: anastomosis between arteria temporalis superficialis and arteria cerebri media $\mathrm{dx}$.

\section{MATERIAL AND METHODS}

Vessel samples of temporal artery (a. temporalis superficialis) were obtained from bilateral bypass surgery and processed by standard histopathological methods. The $5 \mu \mathrm{m}$ thick sections of formalin-fixed paraffin-embedded tissue samples were deparaffinized and hematoxylin-eosin (HE) stained using routine procedures. For visualisation of the internal elastic membrane and/or in elastica stored acid mucopolysaccharides, we used the sorcin-fuchsin and Müller stain method. Immunohistochemical analysis was done using anti-smooth muscle actin (SMA, clone 1A4, diluted $1: 50$, Dako, Denmark), anti-vascular endothelial growth factor (VEGF, A-20, diluted 1:25, Santa Cruz Biotechnology, USA), anti-VEGF receptor (VEGFR, Flk-1/A-3, diluted 1 : 50, Santa Cruz Biotechnology, USA) and anti-nestin (clone 10C2, diluted 1: 200, Chemicon International, USA) antibodies after endogenous peroxidase blocking and antigen retrieval carried out in phosphate-buffered saline solution ( $\mathrm{pH} \mathrm{7.5,} \mathrm{3-times} \mathrm{for} 5 \mathrm{~min}$ ). For antigen imaging, the EnVision kit (Dako, Denmark)
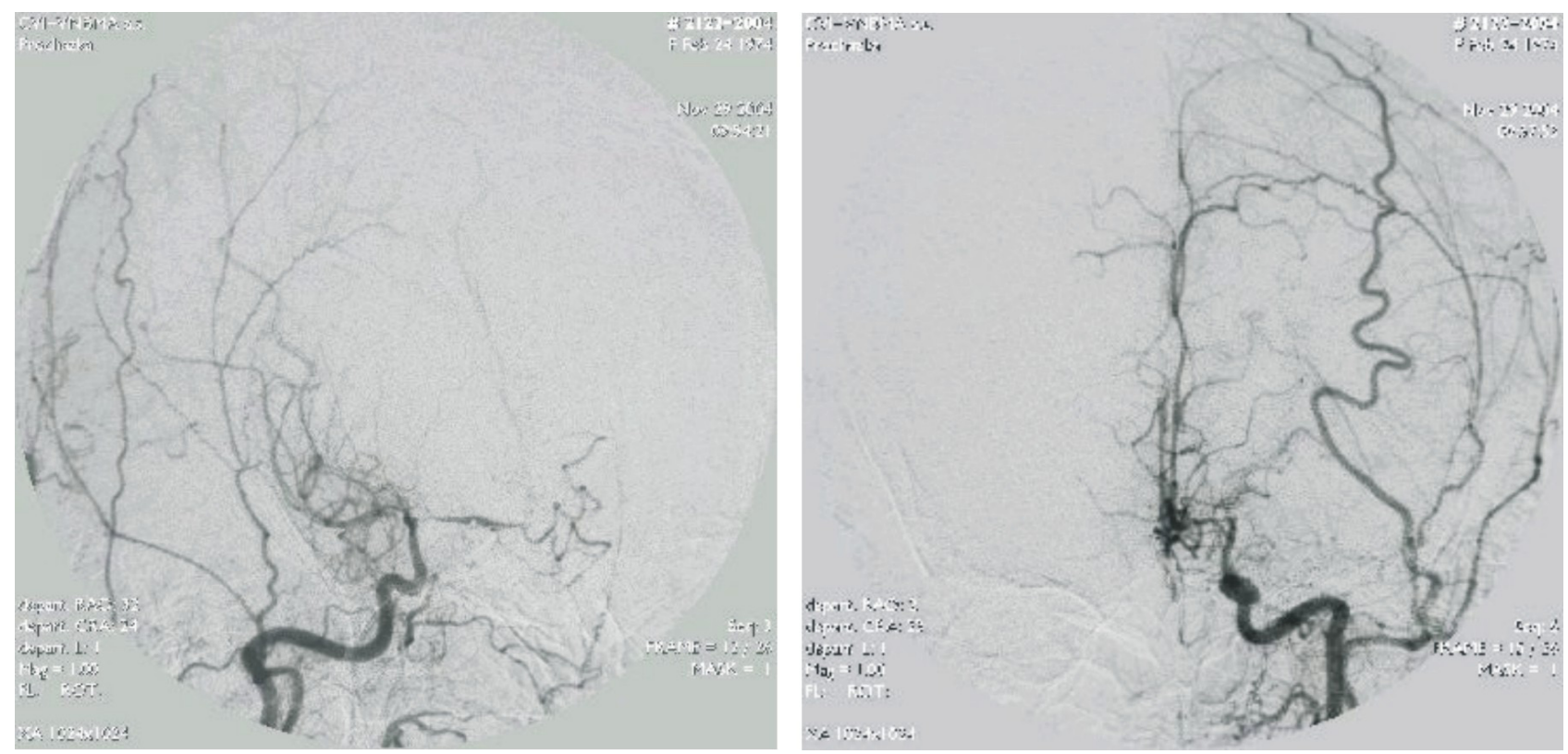

Fig. 1. Preoperative angiography of the right (a) and left (b) arteria carotis communis shows severe stenosis of both internal carotid arteries (arrows) and their bifurcation. Elongated and coiled vessels like ("smoke puffs carried away by breeze") are seen bilaterally. 

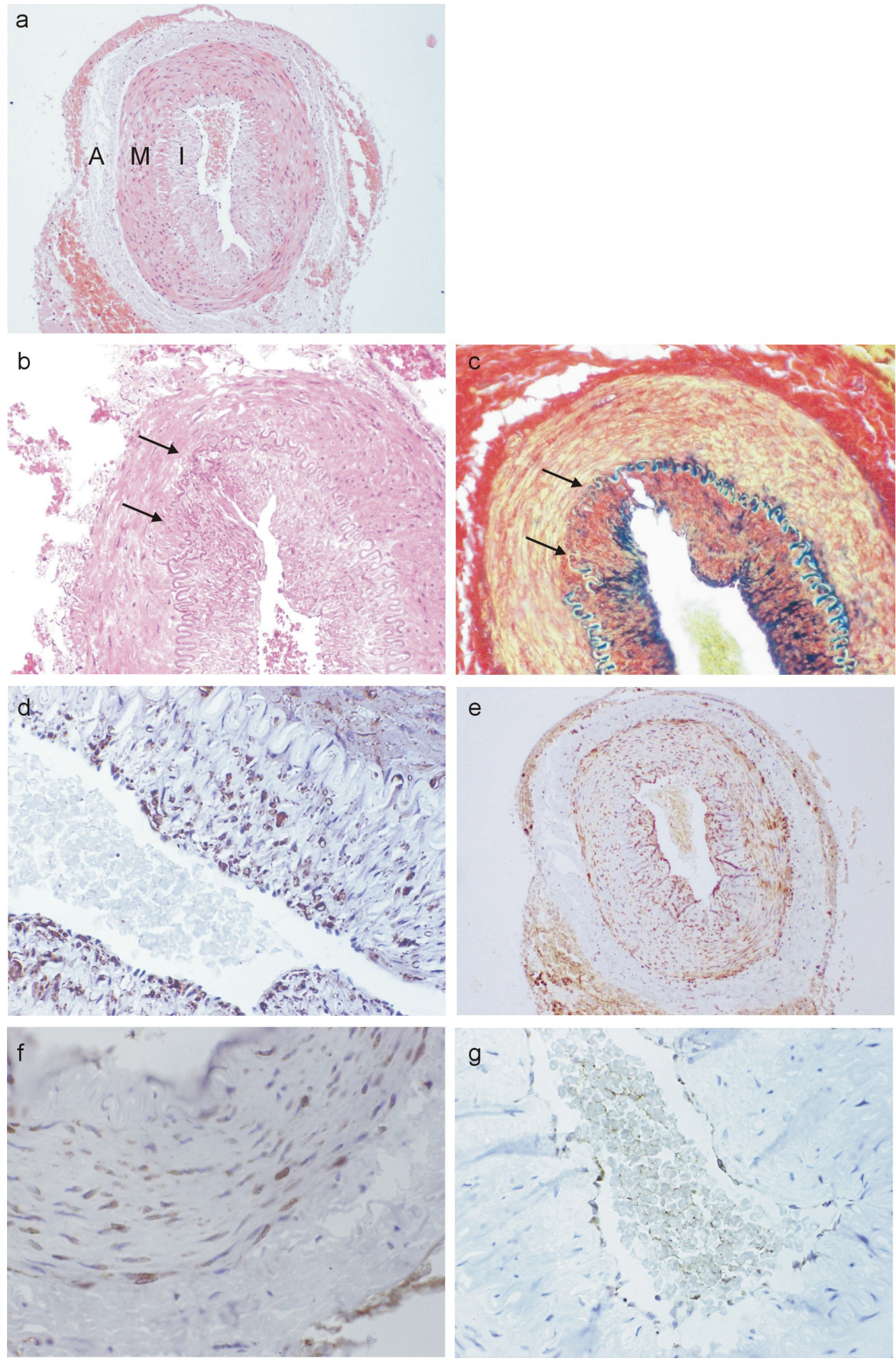

Fig. 2. Microscopical and immunohistochemical features of moyamoya disease (I - intima, M - media, A - adventicia). (a) Stenosis of muscular arteria with thickened intima composed of fibrocellular material without lipid deposits or calcifications, HE, original magnification 100x. (b, c) Extremely tortuosed internal elastic membrane with disruption, elastica stain (b), alcian blue positivity - Müller stain (c), original magnification 200x. (d) Decreased number of smooth muscle cells in media and SMA positivity of the arterial smooth muscle cells promoting the intima thickening, original magnification 400x. (e) The expression of VEGF in endothel, thickened intima and media, original magnification 100x. (f) The positivity of cells in media for VEGFR original magnification 400x. (g) Positive reaction for nestin in endothelial cells, original magnification 400x. 
was used. All sections were conterstained with hematoxylin, dehydrated and mounted. Negative controls for immunostaining included omitting primary antibody, cases of capillary hemangiomas and glioblastomas were used as positive controls.

\section{PATHOLOGICAL FINDINGS}

Microscopic findings of the right temporal artery (Fig. 2a) revealed thickening of intima, irregular undulation of the internal elastic lamina with disruption (Fig. 2b,2c) and attenuation of the media.

The fibrocellular matter of the thickened intima was positive for SMA (Fig.2d.) and VEGF (Fig. 2e). Focal absence of SMA (Fig. 2d) and evident VEGFR (Fig. 2f) as well as VEGF positivity (Fig. 2e) were revealed in the media of examined vessels. The endothelial cells were positive for VEGF (Fig. 2e). Positive reaction for nestin was demonstrated in endothelial cells of dilated or irregular shaped vessels (Fig. 2g).

\section{DISCUSSION}

Data from recent studies have suggested that various immunohistochemical changes in affected arteries can be associated with MMD. Abnormalities in caspase-3 expression in vessel wall have been described as well as increased expression of hepatocyte growth factor (HGF) and its receptor c-Met in the media and thickened intima $^{11,12}$. The latter suggest that enhanced expression of HGF in the arterial smooth muscle cells might promote thickening of the intima and encourage migration of the muscle cells into the intima. Takekawa et al. ${ }^{6}$ hypothesized that VEGF and VEGFR induced by hypoxia might act as regulatory factors for angiogenesis including the abnormal vascular network. However, they found no differences in expression of VEGF/VEGFR between affected and non-affected vessels. This is not in agreement with our findings since we found higher expression of both angiogenetic factors in intima and in the case of VEGF also in the endothelium of moyamoya affected arteries than normal ones. The reason for this discrepancy might be the fact that Takekawa et al. ${ }^{6}$ examined postmortum specimens whereas we examined biopsies. Recently, Jung et al. ${ }^{13}$ described an increase in number and activity of endothelial progenitor cells in the blood of patients with MMD. Nestin is a marker of progenitor and newly formed endothelial cells ${ }^{14}$. Therefore, we included the nestin immunostaining in the panel for the MMD examination and we found that the endothelium of MMD affected vessels is nestin positive. This, together with the finding of VEGF/ VEGFR expression, might suggest an active angiogenetic process in MMD and complete the battery of immunostainings for its diagnosis. Thus, we bring a new suggestion but further studies with higher number of patients is necessary to elucidate the role of these growth factors in the pathogenesis of moyamoya disease.

\section{ACKNOWLEDGEMENT}

This work was supported in part by grant MSM 6198959216.

\section{REFERENCES}

1. Yonekawa Y, Ogata N, Kaku Y, Taub E, Imhof HG. Moyamoya disease in Europe, past and present status. Clin Neurol Neurosurg 1997; 99:S58-60.

2. Kuroda S, Houkin K. Moyamoya disease: current concepts and future perspectives. Lancet Neurol 2008; 7:1056-1066.

3. Suzuki J, Takaku A. Cerebrovascular „moyamoya“ disease. Disease showing abnormal net-like vessels in base of brain. Arch Neurol 1969; 20:288-99.

4. Kuroda S, Houkin K. Moyamoya disease: current concepts and future perspectives. Lancet Neurol 2008; 7:1056-66.

5. Yamashita M, Oka K, Tanaka K. Histopathology of the brain vascular network in moyamoya disease. Stroke 1983; 14:50-8.

6. Takekawa Y, Umezawa T, Ueno Y, Sawada T, Kobayashi M. Pathological and immunohistochemical findings of an autopsy case of adult moyamoya disease. Neuropathology 2004; 24:236-42.

7. Yanagawa Y, Sugiura T, Suzuki K, Okada Y. Moyamoya disease associated with positive findings for rheumatoid factor and myeloperoxidase-anti-neutrophil cytoplasmic antibody. West Indian Med J 2007; 56:282-84.

8. Kim SJ, Heo KG, Shin HY, Bang OY, Kim GM, Chung CS, Kim $\mathrm{KH}$, Jeon P, Kim JS, Hong SC, Lee KH. Association of thyroid autoantibodies with moyamoya-type cerebrovascular disease: a prospective study. Stroke 2010; 41:173-176.

9. Yamada H, Deguchi K, Tanigawara Y, et al. The relationship between moyamoya disease and bacterial infection. Clin Neuro Neurosurg 1997; 99: S221-4.

10. Mineharu Y, Takenaka K, Yamakawa T, Inoue K, Ikeda H, Kikuta $\mathrm{KI}$, et al. Inheritance pattern of familial moyamoya disease: autosomal dominant mode and genomic imprinting. J Neurol Neurosurg Psychiatry 2006; 77: 1025-9.

11. Takagi Y, Kikuta K, Sadamasa N, Tanaka K. Caspase-3-dependent apoptosis in middle cerebral arteries in patients with moyamoya disease. Neurosurgery 2006; 59: 894-900.

12. Nanba R, Kuroda S, Ishikawa T, Houkon K, Iwasaki Y. Increased expression of hepatocyte growth factor in cerebrospinal fluid and intracranial artery in moyamoya disease. Stroke 2004; 35:2837-42.

13. Jung KH, Chu K, Lee ST, Park HK, Bahn JJ, Kim DH, et al. Circulating endothelial progenitor cells as a pathogenetic marker of moyamoya disease. J Cereb Blood Flow Metab 2008; 11:17951803.

14. Mokry J, Cizkova D, Filip S, Ehrmann J, Osterreicher J, Kolar Z, English D. Nestin expression by newly formed human blood vessels. Stem Cell Dev 2004; 13:658-64. 